\title{
Du trağ́que
}

en médecine

Jacques Quintin

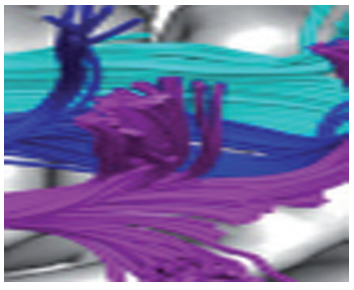

Bureau de développement de l'éthique, Faculté de médecine et des sciences de la santé, Université de Sherbrooke, 3001 , $12^{\mathrm{e}}$ avenue nord, Sherbrooke (Québec), Canada. jacques.quintin@usherbrooke.ca

II tombe sous le sens qu'une pratique de triage fait partie inhérente de la médecine pour offrir de meilleurs soins. Ce qui se montre avec moins d'évidence, ce sont tous les enjeux médicaux, sociaux, économiques et éthiques qui se rattachent à une médecine du tri. Au début du XIX ${ }^{e}$ siècle s'est établi un mécanisme de tri afin d'orienter les malades, soit vers les hôpitaux, soit vers les hospices. Le critère principal pour départager les malades fut celui de la curabilité. Pour avoir un accès à l'hôpital, donc à des soins médicaux, il ne fallait pas être trop malade. Dans ce nouveau contexte hospitalier, les malades recevaient des soins afin de pouvoir retrouver leur capacité à travailler. Nous voyons que le concept de rétablissement, aujourd'hui comme hier, se situe entre la pensée médicale et la raison administrative. Ces deux ordres du discours ne sont pas si étanches qu'on pourrait le laisser croire. Le livre de Guillaume Lachenal, Céline Lefève et Vinh-Kim Nguyen montre qu'il existe une collusion entre les critères médicaux, sociaux, économiques et moraux [1]. Le triage requiert de définir des priorités dans l'utilisation des ressources limitées. Cette définition des priorités

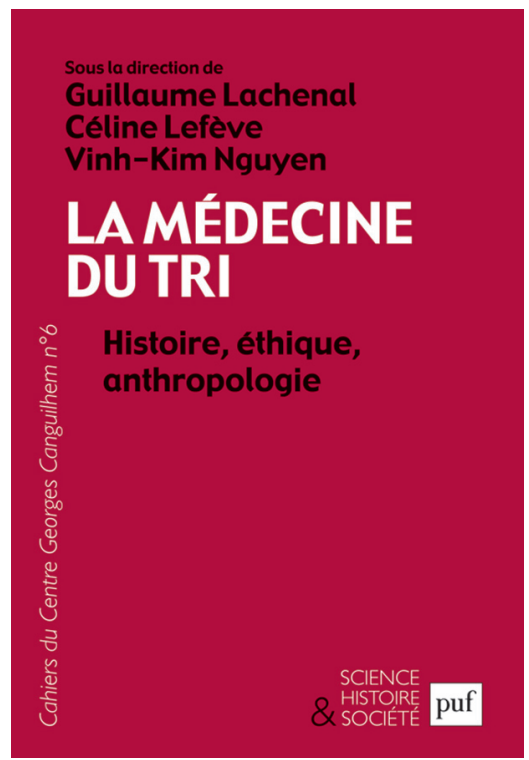

contrario, que fait-on malgré la futilité des soins, si la vie continue d'avoir un sens pour le malade? Est-ce que survivre est une valeur en soi ? La ligne de démarcation entre avantages et inconvénients d'un soin est brouillée du fait que «ce que les patients valorisent dans la qualité de vie diffèrent de ce que les médecins valorisent ». Il existe ainsi un écart important entre des critères médicaux ou pragmatiques et des critères existentiels.

Si la médecine du tri a pris son essor dans un contexte d'urgence, principalement celui du champ de bataille propre à la Première Guerre mondiale, on assiste aujourd'hui à une extension de cette pratique du triage, de sorte que les enjeux ne sont pas identiques. II existe une différence entre exercer une médecine d'urgence envers des blessés graves selon des critères médicaux, dont celui de la survie, et une médecine de catastrophe où on assiste à un renversement des priorités de sorte qu'on tentera de sauver le plus grand nombre de malades possible en privilégiant les plus aptes à survivre, donc les moins malades, selon des critères utilitaristes. Dans le premier contexte, on se demande qui est le plus gravement atteint, tandis que dans le second contexte, on s'interroge sur «qui sauver quand on ne peut pas sauver tout le monde ». Bref, les enjeux éthiques ne sont pas les mêmes. Les médecins sont pris entre appliquer des critères médicaux et des critères qui relèvent de l'égalitarisme ou de l'utilitarisme. Peu importe les critères, le médecin est confronté au fait de choisir entre la vie et la mort: plus exactement de donner la vie ou la mort. Ce ne va pas sans une prise en compte des moyens médicaux à notre disposition, et sans un jugement de valeur sur la vie des malades. La question consiste à savoir s'il est « futile de produire des formes de vie diminuée ». Autrement dit, que signifie une vie sauvée physiologiquement si cette même vie ne revêt plus de sens pour le malade ? Ou, a rôle de souverain, le médecin l'assume difficilement. De plus, en situation de catastrophe, le triage implique «le passage, pour le meilleur et le pire, d'une norme de justice distributive à une autre ».

Nous voyons que l'exercice de la médecine est un geste social et politique qui engage d'autres critères que les critères médicaux et d'autres experts que les médecins et les autorités morales. Dans un tel contexte, où sont 
interpellés des enjeux éthiques, il est de mise que la réflexion ne soit pas réservée qu'aux seuls médecins; d'autant plus que l'impartialité des médecins et des humanitaires n'est pas si étanche qu'on le croit. La complexité des situations rencontrées exigent certes des prises de décisions qui reposent sur des raisons médicales. Mais elles engagent aussi d'autres logiques, d'autres valeurs et d'autres idéologies, de sorte que, parfois, les émotions peuvent jouer un rôle crucial dans les prises de décisions. II y a des malades qui recevront plus de soins parce que les professionnels de la santé se sentent davantage touchés par la situation. Les médecins sont aussi mus par l'émotion «sauver à tout prix », qui n'est pas toujours très efficace d'un point de vue administratif. Si nous appliquons la distinction établie par Weber entre les éthiques de la conviction et de la responsabilité, nous constatons que les médecins sont écartelés entre le respect de leur devoir ou de leur conviction et le souci d'être efficace selon les conséquences de leur geste ou de leur pronostic. Il y a donc aussi les différentes logiques administratives. Par exemple : « Faut-il soigner les touristes? », question qui remet en cause le «principe de l'accès inconditionnel aux soins », de sorte qu'il n'est plus «possible de penser le soin sans sa prise en charge financière ». Cela implique la mise en place de critères d'exclusion qui compromettent les principes de solidarité.

Ce collectif d'auteurs a le mérite de présenter une approche multidisciplinaire qui donne une excellente description des différentes pratiques de triage. Comme il est écrit majoritairement par des médecins, des historiens et des anthropologues, le contraire aurait été surprenant. Mais si nous ne voulons pas reproduire le raisonnement fallacieux dénoncé par Hume [2]1' à savoir prescrire le cours de l'action en raison de ce qui existe déjà, il conviendrait de reprendre ce sujet du tri à l'aide de concepts prescriptifs. Ce qui devrait être ne relève pas de ce qui est, mais de notre manière d'imaginer la vie selon nos souhaits. Si le rationnement est inévitable, il n'en demeure pas moins qu'une réflexion élargie sur les enjeux entourant la médecine du tri est aussi nécessaire. Cette réflexion ne peut se faire sans l'implication des différents acteurs que sont les médecins, les patients, les proches et les institutions, pour parvenir à un accord sur les « bonnes » raisons d'agir. Reste maintenant à entendre la voix du public. II ne s'agit pas de dialoguer parce qu'on penserait que le dialogue est le meilleur outil pour la résolution de problème complexe. Il convient de s'exercer au dialogue pour faire ressortir nos propres présupposés qui guident nos choix et nos réflexions. C'est un enjeu éthique, mais surtout philosophique, dans la mesure où il s'agit de justifier nos choix. Cela engage deux questions fondamentales : dans quel type de société désirons-nous vivre? Et quel sens accordons-nous à l'existence humaine?

Il existe une autre menace que celle de notre survie, c'est celle qui découle des pratiques qui ignorent le poids du sens des choses vécu par le public. Peu importe le choix des critères ou du système de normes qui nous permettrait de s'entendre sur les pratiques du triage en médecine, il n'en demeure pas moins, si ces critères sont utilisés comme des fin en soi et non comme des moyens, que nous oublierons qu'une personne malade, c'est une personne vivante portée par une histoire de vie singulière, et non pas une simple histoire de cas ou un dossier. Ce que l'on vise idéalement à tra-

${ }^{1}$ David Hume (7 mai 1711 - 25 août 1776) est un philosophe, économiste et historien britannique. vers l'établissement de critères ou d'un système de normes, c'est de répondre de manière créative à des situations d'exception. Pour cette raison, il convient d'introduire un peu - ou beaucoup - de subjectivité pour interpréter et transgresser ces critères sur lesquels pourtant on s'entend. C'est tout l'art médical ou l'art du discernement, comme quoi la médecine ne peut pas se permettre une économie de la réflexion philosophique sur ce que l'on est en train de faire et sur ce que l'on pense être le mieux.

Le pire scénario serait, pour des médecins et des administrateurs, de ne jamais remettre en question les critères sur lesquels ils se reposent pour justifier leur choix. Le second scénario inacceptable serait de croire que la remise en question relèverait du seul apanage des dominants, c'est-à-dire les instances médico-scientifiques et administratives. La médecine du tri est devenue plus complexe que celle qui s'exerçait sur le champ de bataille. Cela implique aussi une résolution de problèmes plus complexes en incluant la prise en compte de la voix des malades, des proches et du public, et en n'oubliant pas la visée du triage : sauver des vies sans savoir avec certitude lesquelles. Nous sommes en pleine tragédie : accomplir l'horreur pour participer à un peu de bonheur pour quelques-uns. Encore faut-il être de bonne volonté, ce qui, dans un autre contexte, par exemple celui des camps d'extermination nazis, n'était sûrement pas le cas. En revanche, l'un dans l'autre, comme le proposent les directeurs de ce collectif, on doit exercer une critique du tri : comment se fait-il que nous sommes pris à exercer une médecine du tri ? Ou encore : ne sommesnous pas tous responsables des conditions qui rendent la médecine du tri nécessaire? Que nous adoptions une perspective médicale, utilitariste ou égalitariste, cela ne change pas la question de fond : la question des conditions de possibilité pragmatiques ou des présupposés, question philosophique par excellence. Ce faisant, devant des pratiques et des critères, même éthiques, s'ils heurtent notre sensibilité, il y a tout lieu de les interpréter au lieu de se dissoudre dans un ramassis de connaissances en forme de mosaïque atomique. Pour cela, il convient de déplacer notre attention pour comprendre autrement. $\diamond$

The tragic side of medicine

\section{LIENS D'INTÉRÊT}

L'auteur déclare n'avoir aucun lien d'intérêt concernant les données publiées dans cet article.

\section{RÉFÉRENCES}

1. Lachenal G, Levève C, Nguyen VK. La médecine du tri. Histoire, éthique, anthropologie. Paris : PUF, 2014 : 312 p.

2. Hume D. La morale. Traité de la nature humaine III. Paris : GF-Flammarion, $1993: 65$.

\section{TIRÉS À PART}

J. Quintin 